\title{
Increased arterial stiffness in patients with chronic obstructive pulmonary disease: a mechanism for increased cardiovascular risk
}

\author{
N L Mills, ${ }^{1} \mathrm{~J} J$ Miller, ${ }^{2}$ A Anand, ${ }^{1}$ S D Robinson, ${ }^{1}$ G A Frazer, ${ }^{2}$ D Anderson, ${ }^{2}$ L Breen, ${ }^{3}$ \\ I B Wilkinson, ${ }^{4}$ C M McEniery, ${ }^{4} \mathrm{~K}$ Donaldson, ${ }^{2}$ D E Newby, ${ }^{1}$ W MacNee ${ }^{2}$
}

${ }^{1}$ Centre for Cardiovascular Science, University of Edinburgh, Edinburgh, UK; ${ }^{2}$ ELEGI Colt Research Laboratories, University of Edinburgh, Edinburgh, UK; ${ }^{3}$ Clinical Research Facility, Royal Infirmary Edinburgh, Edinburgh, UK;

${ }^{4}$ Clinical Pharmacology Unit, University of Cambridge, Cambridge, UK

Correspondence to: Dr J J Miller, ELEGI-Colt Laboratory, Queen's Medical Research Institute, The University of Edinburgh, 47 Little France Crescent, Edinburgh EH16 4TJ, UK:

joy.miller@ed.ac.uk

NLM and JJM contributed equally to this paper.

Received 28 April 2007 Accepted 24 October 2007 Published Online First 16 November 2007

\section{ABSTRACT}

Rationale: Chronic obstructive pulmonary disease (COPD) is associated with a 2-3-fold increase in the risk of ischaemic heart disease, stroke and sudden death. The mechanisms responsible for this association are not clear and appear to be independent of smoking history.

Objective: We test the hypothesis that patients with COPD have increased arterial stiffness and blood pressure in comparison with age and smoking matched controls.

Methods: In a prospective case control study, we recruited 102 patients with COPD and 103 healthy controls matched for age and smoking status. Patients were assessed by clinical history and spirometry, with arterial stiffness and blood pressure determined using radial artery applanation tonometry and sphygmomanometry.

Results: Patients with COPD had increased arterial stiffness compared with matched controls, with elevated augmentation pressure (17 (1) vs 14 (1) mm Hg; $p=0.005)$ and a reduced time to wave reflection (131 (1) vs 137 (2) $\mathrm{ms} ; \mathrm{p}=0.004$ ). These differences were associated with increases in both diastolic (82 (1) vs 78 (1) $\mathrm{mm} \mathrm{Hg} ; \mathrm{p}=0.005$ ) and systolic blood pressure (147 (2) vs 132 (2) $\mathrm{mm} \mathrm{Hg} ; \mathrm{p}<0.001$ ). Serum $\mathrm{C}$ reactive protein concentrations were threefold higher in patients (6.1 (0.9) vs 2.3 (0.4) mg/l; $p=0.001$ ). Data are presented as mean (SEM).

Conclusions: Patients with COPD have increased arterial stiffness and blood pressure in comparison with controls matched for age and smoking status. We speculate that increased systemic inflammation and vascular dysfunction could potentially explain the excess cardiovascular morbidity and mortality associated with COPD.

Although primarily a lung disease, chronic obstructive airways disease (COPD) is now recognised to have important systemic consequences that may affect morbidity and mortality. ${ }^{1}$ In particular, it is associated with a markedly increased risk of cardiovascular disease. ${ }^{2}$ Observational studies suggest that reduced expiratory flow volume (forced expiratory volume in $\left.1 \mathrm{~s}\left(\mathrm{FEV}_{1}\right)\right)$, a characteristic feature of COPD, is associated with a $\sim 3$-fold increase in the risk of ischaemic heart disease, stroke and sudden death, with cardiovascular mortality accounting for up to $50 \%$ of all deaths in patients with COPD. ${ }^{34}$ While most patients with COPD are current or ex-smokers, this increased cardiovascular risk is independent of cigarette smoking habit. ${ }^{25}$

The mechanisms of increased cardiovascular risk in COPD remain poorly understood. COPD is characterised by excessive pulmonary inflammation in response to cigarette smoking and air pollution. ${ }^{6}$ However, it is increasingly recognised that COPD is also associated with a systemic inflammatory response. ${ }^{7}$ During the past 10 years, the role of inflammation and oxidative stress in the pathogenesis of atherosclerosis has been established. ${ }^{8}$ The inflammatory mediator $\mathrm{C}$ reactive protein (CRP) is an important predictor of cardiovascular outcome in patients with and without coronary artery disease, and has been directly implicated in the pathogenesis of atherosclerotic plaque formation. CRP is increased along with a variety of acute phase proteins in patients with COPD, ${ }^{9}$ and may contribute to the development and clinical complications of atherosclerosis in these patients.

Atherosclerosis is a disease of the large and medium sized elastic arteries in which one of the earliest pathological features is endothelial dysfunction. ${ }^{10}$ The vascular endothelium plays an important role in the maintenance of vascular tone through the local release of vasoactive compounds such as nitric oxide. With increasing age and in the presence of cardiovascular risk factors, vascular dysfunction contributes to reduced arterial compliance and increased central arterial pressure. ${ }^{11}$ Increased large artery stiffness results in greater central aortic systolic pressures, increased left ventricular afterload and reduced diastolic coronary artery filling. Arterial stiffness may be the central pathological process involved in essential hypertension and is an independent predictor of cardiovascular mortality in these patients. ${ }^{12}$

Applanation tonometry of the radial artery and pulse wave analysis can be used to derive measures of arterial stiffness and central aortic blood pressure. Previously, central arterial stiffness has been linked to reduced $\mathrm{FEV}_{1}$ in apparently healthy men without coronary heart disease. ${ }^{13}$ Increased central arterial stiffness and blood pressure in patients with COPD may explain the excess cardiovascular morbidity and mortality in these patients. In a prospective case control study, we assessed arterial stiffness and blood pressure in patients with COPD and controls matched for age and current smoking status.

\section{METHODS \\ Subjects}

Patients with COPD were recruited through general practice databases and a specialised outpatient clinic at the Royal Infirmary of Edinburgh, 


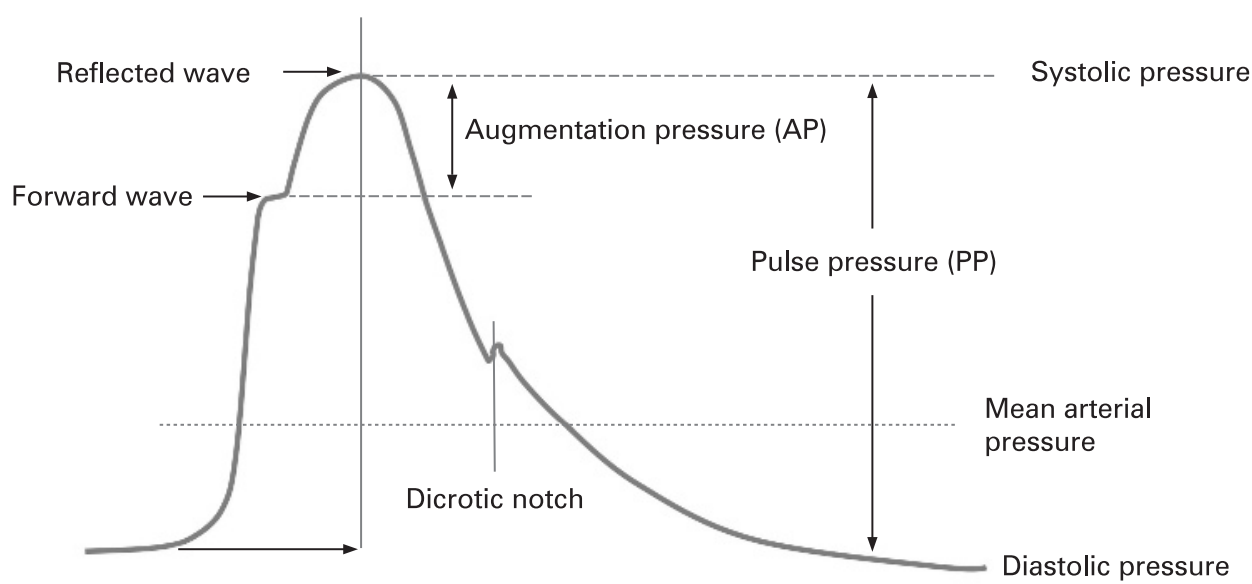

Time to wave reflection $(\mathrm{Tr})$

Figure 1 An aortic pulse waveform as produced by the SphygmoCor system from applanation tonometry of the radial artery. Augmentation pressure is the difference between the systolic peak (forward wave) and first systolic inflection (reflected wave) pressures. This difference divided by the pulse pressure generates the augmentation index. Adapted from Smith et al 2000. ${ }^{35}$

Edinburgh, UK. COPD was defined by European Respiratory Society/American Thoracic Society guidelines. ${ }^{14}$ Patients with a recent exacerbation (within 6 weeks) were excluded. Patients with a history of asthma, bronchiectasis, pulmonary fibrosis, pneumonectomy or pulmonary lobectomy, rheumatoid arthritis, inflammatory bowel disease, connective tissue disorders or malignancy within the previous 5 years and those treated with long term steroids, oral theophylline or immunosuppressive therapy were excluded. Healthy controls were recruited through primary care and were not taking any regular medication. Cases and controls were matched by frequency for age and current smoking status using the mean values for each group. All studies were performed with local ethics approval, written informed consent of all subjects and in accordance with the Declaration of Helsinki.

\section{Study design}

In a prospective case control study, we recruited 102 patients with COPD, and 103 healthy controls matched for age and current smoking status. Subjects were asked to refrain from smoking for $12 \mathrm{~h}$ before assessment. Prior to assessment, all medications were withheld for at least $12 \mathrm{~h}$, with long acting

Table 1 Baseline characteristics of patients with chronic obstructive pulmonary disease and matched healthy controls

\begin{tabular}{lccl}
\hline & $\begin{array}{l}\text { Healthy } \\
\text { controls } \\
(\mathbf{n}=103)\end{array}$ & $\begin{array}{l}\text { COPD } \\
\text { patients } \\
(\mathbf{n}=102)\end{array}$ & p Value \\
\hline Sex (M/F) & $51 / 52$ & $61 / 41$ & 0.161 \\
Age (y) & $66.7(0.7)$ & $67.1(0.7)$ & 0.677 \\
Current smoker & $49(48)$ & $48(47)$ & 1.000 \\
Ex-smoker & $54(52)$ & $54(53)$ & 1.000 \\
Serum total cholesterol (mmol/) & $5.5(0.1)$ & $5.7(0.1)$ & 0.329 \\
LDL cholesterol (mmol/) & $3.2(0.1)$ & $3.1(0.1)$ & 0.444 \\
HDL cholesterol $(\mathrm{mmol} /)$ & $1.5(0.1)$ & $1.6(0.1)$ & 0.335 \\
C reactive protein $(\mathrm{mg} / \mathrm{l})$ & $2.3(0.4)$ & $6.1(0.9)$ & 0.001 \\
Height $(\mathrm{cm})$ & $168(0.9)$ & $164(2.3)$ & 0.097 \\
Weight $(\mathrm{kg})$ & $76(1.3)$ & $69(2.2)$ & 0.005 \\
Body mass index $\left(\mathrm{kg} / \mathrm{m}^{2}\right)$ & $27(0.4)$ & $25(0.7)$ & 0.014 \\
\hline
\end{tabular}

Values are mean (SEM) or number (\%).

Significance (p): unpaired t test (continuous variables), $\chi^{2}$ (categorical data).

COPD, chronic obstructive pulmonary disease; HDL, high density lipoprotein; LDL, low density lipoprotein. anticholinergic treatment withheld for $24 \mathrm{~h}$. A subgroup of COPD patients with no history of cardiovascular comorbidities was identified. From the history and medical records this group had no prior diagnosis of, and were not on medication for, ischaemic heart disease, hypertension, hypercholesterolaemia or diabetes mellitus. Using the same criteria, control subjects were screened for these cardiovascular risk factors prior to inclusion.

\section{Pulse wave analysis}

Vascular studies were performed in a quiet, temperature controlled room with subjects resting in the supine position. Systolic and diastolic blood pressures were measured in duplicate using a semi-automated non-invasive oscillometric sphygmomanometer, following a $10 \mathrm{~min}$ rest period. Pulse wave analysis was performed using micromanometer (Millar Instruments, Texas, USA) applanation tonometry of the radial artery at the wrist and the SphygmoCor system (AtCor Medical, Sydney, Australia), in accordance with the manufacturer's recommendations. Briefly, pulse wave analysis derives an aortic pulse pressure waveform from the radial artery wave via a mathematical transfer function. The arterial pressure waveform is a composite of the forward pressure wave created by ventricular contraction and a reflected wave generated by peripheral vascular resistance (fig 1)..$^{15}$ The augmentation index, which is the difference between the second and first systolic peaks expressed as a percentage of the pulse pressure, is a measure of systemic arterial stiffness and wave reflection. The time to wave reflection $(\operatorname{Tr})$ is reduced with increasing arterial stiffness, and provides a surrogate of aortic pulse wave velocity. ${ }^{16}$

At least two independent waveform analyses were obtained from each subject, with measurements only accepted on meeting SphygmoCor quality control criteria. These replicate measures of augmentation pressure were highly correlated across the study population $(\mathrm{r}=0.939, \mathrm{p}<0.0001, \mathrm{n}=205)$. Measurements were performed by staff specifically trained in the technique and blinded to the clinical characteristics of each subject.

\section{Assays}

Differential white blood cell count and platelets were determined using an autoanalyser. Serum lipid profile was determined by the regional accredited clinical biochemistry 
Table 2 Baseline characteristics of patients with chronic obstructive pulmonary disease with and without established cardiovascular comorbidities

\begin{tabular}{lccl}
\hline & $\begin{array}{l}\text { COPD with CV } \\
\text { comorbidity } \\
\text { (n= 47) }\end{array}$ & $\begin{array}{l}\text { COPD without } \\
\text { CV comorbidity } \\
\text { (n = 55) }\end{array}$ & p Value \\
\hline Sex (M/F) & $28 / 19$ & $33 / 22$ & 1.00 \\
Age (y) & $67.3(1.0)$ & $66.9(1.0)$ & 0.217 \\
Current smoker & $19(40)$ & $29(53)$ & 0.238 \\
Ex-smoker & $28(60)$ & $26(47)$ & 0.238 \\
Serum total cholesterol (mmol/l) & $5.5(0.2)$ & $5.8(0.1)$ & 0.118 \\
LDL cholesterol (mmol/l) & $2.9(0.2)$ & $3.2(0.2)$ & 0.376 \\
HDL cholesterol $(\mathrm{mmol} / \mathrm{l})$ & $1.4(0.1)$ & $1.7(0.1)$ & 0.670 \\
C reactive protein $(\mathrm{mg} / \mathrm{l})$ & $6.8(1.7)$ & $5.4(0.7)$ & 0.115 \\
Height $(\mathrm{cm})$ & $162(3.0)$ & $165(3.4)$ & 0.982 \\
Weight $(\mathrm{kg})$ & $70(3.2)$ & $69(3.0)$ & 0.756 \\
Body mass index $\left(\mathrm{kg} / \mathrm{m}^{2}\right)$ & $26(1.1)$ & $24(0.8)$ & 0.092 \\
\hline
\end{tabular}

Values are mean (SEM) or number (\%).

Significance $(p)$ unpaired $t$ test or $\chi^{2}$.

$\mathrm{COPD}$, chronic obstructive pulmonary disease; CV, cardiovascular; HDL, high density lipoprotein; LDL, low density lipoprotein.

laboratory. Serum CRP concentrations were measured using a highly sensitive immunonephelometric assay (Behring BN II nephelometer, Marburg, Germany).

\section{Data analysis and statistics}

Data were analysed using SPSS (V.14). Comparisons were made between patients with COPD and matched controls using unpaired $t$ tests for continuous variables and $\chi^{2}$ analysis for categorical data. Comparisons were made between controls and the two pre-specified COPD subgroups, those patients with or without a history of cardiovascular comorbidities, using analysis of variance (ANOVA). Correlations between hsCRP and measures of arterial stiffness were analysed in patients with COPD and controls using the Pearson's correlation coefficient. Continuous variables were reported as mean (SEM) and statistical significance was taken at $\mathrm{p}<0.05$.

\section{RESULTS}

The populations were matched for age and current smoking status (table 1). In patients with COPD, mean post-bronchodilator
$\mathrm{FEV}_{1}$ was 46 (2)\% predicted, post-bronchodilator FVC 68 (2)\% predicted and $\mathrm{FEV}_{1} / \mathrm{FVC}$ ratio 50 (1)\% predicted. When stratified by Global Initiative for Chronic Obstructive Lung Disease (GOLD) stage, $2 \%$ of patients were stage I, $36 \%$ stage II, $39 \%$ stage III and $20 \%$ stage IV. While well matched for current smoking status, there was a modest yet significant difference between patients and controls (46 (2) vs $33(2)$ pack years; $p=0.004)$. Weight $(p=0.005)$ and body mass index $(p=0.014)$ were reduced in patients with COPD compared with healthy controls. In patients with COPD, lipid profiles were similar in the presence or absence of established cardiovascular comorbidities. Serum CRP concentrations were higher in the COPD groups than in controls ( $p=0.001)$.

The two pre-specified COPD subgroups, those patients with or without a history of cardiovascular comorbidities, were well matched (table 2). Patients with COPD had increased arterial stiffness compared with matched controls (table 3), with elevated augmentation pressures (17 (1) vs 14 (1) $\mathrm{mm} \mathrm{Hg}$; $\mathrm{p}=0.015$ ) (fig 2) and a reduced time to wave reflection (131 (1) vs 137 (2) ms; p=0.005) (fig 3). These relationships remained significant in the COPD subgroup with no history of cardiovascular comorbidities. Consistent with increased arterial stiffness, these differences were associated with increases in both systolic (147 (2) vs 132 (2) mm Hg; p <0.001) and diastolic (82 (1) vs 78 (1) mm Hg; p = 0.007) blood pressure. Serum CRP concentrations were threefold higher in patients than in controls (6.0 (0.9) vs 2.3 (0.4) $\mathrm{mg} / \mathrm{l} ; \mathrm{p}=0.001$ ) (fig 4). Serum CRP concentrations correlated with augmentation pressure in patients with COPD $(r=0.736, p=0.034)$ but not in controls $(r=-0.154, p=0.21)$.

\section{DISCUSSION}

Patients with COPD have increased arterial stiffness, blood pressure and systemic inflammation in comparison with controls matched for age and current smoking status. These differences are not caused by the presence of comorbidity in patients with COPD as arterial stiffness and pressure measurements were similar in the subgroup of patients without existing ischaemic heart disease, hypertension, hypercholesterolaemia or diabetes mellitus. These findings provide a plausible mechanism for the increased cardiovascular morbidity and mortality associated with COPD.

Table 3 Arterial stiffness and blood pressure in patients with chronic obstructive pulmonary disease and matched healthy controls

\begin{tabular}{|c|c|c|c|c|}
\hline & $\begin{array}{l}\text { Healthy } \\
\text { controls } \\
(n=103)\end{array}$ & $\begin{array}{l}\text { COPD with CV } \\
\text { comorbidity } \\
\text { (n=47) }\end{array}$ & $\begin{array}{l}\text { COPD without } \\
\text { CV comorbidity } \\
(\mathrm{n}=55)\end{array}$ & p Value \\
\hline Heart rate (bpm) & $67(1)$ & $69(2)$ & $69(2)$ & 0.621 \\
\hline Peripheral sBP (mm Hg) & $132(2)$ & $149(3.5)$ & $146(3)$ & 0.000 \\
\hline Peripheral dBP (mm Hg) & $78(1)$ & $82(2)$ & $83(2)$ & 0.019 \\
\hline Peripheral MAP (mm Hg) & $96(1)$ & $105(2)$ & $104(2)$ & 0.000 \\
\hline Peripheral PP (mm Hg) & $54(1)$ & $68(3)$ & $63(2)$ & 0.000 \\
\hline Central sBP (mm Hg) & $123(2)$ & $137(3)$ & $134(3)$ & 0.000 \\
\hline Central dBP (mm Hg) & $79(1)$ & $83(2)$ & $84(2)$ & 0.011 \\
\hline Central MAP (mm Hg) & $99(1)$ & $105(2)$ & $104(2)$ & 0.045 \\
\hline Central PP $(\mathrm{mm} \mathrm{Hg})$ & $45(1)$ & $54(3)$ & $51(2)$ & 0.001 \\
\hline Augmentation pressure $(\mathrm{mm} \mathrm{Hg})$ & $14(1)$ & $17(1)$ & $16(1)$ & 0.016 \\
\hline Augmentation index $(\%)$ & $30(1)$ & $31(2)$ & $31(1)$ & 0.628 \\
\hline Time to reflection (ms) & $137(2)$ & $131(2)$ & $131(2)$ & 0.015 \\
\hline
\end{tabular}

Values are mean (SEM).

Groups compared with ANOVA.

$\mathrm{COPD}$, chronic obstructive pulmonary disease; $\mathrm{dBP}$, diastolic blood pressure; MAP, mean arterial pressure; $\mathrm{PP}$, pulse pressure; $\mathrm{sBP}$, systolic blood pressure. 


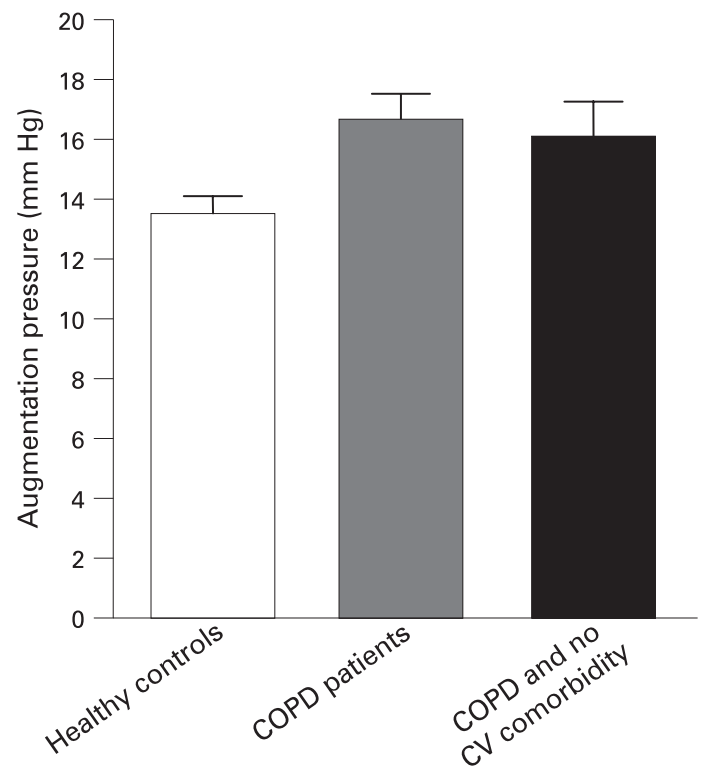

Figure 2 Mean augmentation pressure, a measure of arterial stiffness, was greater in patients with chronic obstructive pulmonary disease (COPD) $(p=0.005)$ and the subgroup of patients with COPD and no cardiovascular (CV) comorbidity $(p=0.04)$ compared with healthy controls matched for age and current smoking status.

\section{Arterial stiffness}

Increased large artery stiffness in patients with COPD was associated with higher peripheral systolic and diastolic blood pressures. While a number of factors may influence arterial tone and blood pressure in these patients, it is possible that increased large artery stiffness is the major determinant of increased blood pressure and hence cardiovascular risk in patients with COPD. Recently it has been established that measures of arterial stiffness, including augmentation and central pulse pressure, independently predict adverse clinical outcome in patients with hypertension. ${ }^{17}$

Arterial stiffness is influenced by both structural and functional aspects of the conduit arteries and resistance beds. Atherosclerosis and calcification of the large arteries decreases vascular compliance, and structural changes in the vessel wall may explain the increased arterial stiffness observed in patients with COPD. Arterial stiffness and calcification increases with age. The average age of our patients was 67 years and yet augmentation pressures were similar to those of a healthy group of 80 year olds, suggesting that COPD may result in premature ageing of the vasculature. ${ }^{11}$ Augmentation pressures in patients with COPD were greater than those of a similar aged population with documented coronary artery disease. ${ }^{18}$ Consistent with this hypothesis, cigarette smoking and impaired lung function are associated with premature ageing and morphological abnormalities in the dermis with reduced elastic fibre content. ${ }^{19}$ It is possible that excessive neutrophil elastase activity in cigarette smokers and patients with $\mathrm{COPD}^{520}$ results in consumption of elastic fibres in the media of the large arteries leading to arterial stiffness.

Arterial stiffness is also determined by the functional properties of the vessel wall with endothelium dependent vasomotor tone involved in the dynamic modulation of augmentation pressure. ${ }^{21} 22$ There is extensive evidence of endothelial dysfunction in cigarette smokers and in patients with atherosclerosis. $^{23}{ }^{24}$ Whether COPD causes an additional impairment of systemic endothelial dysfunction is not yet known. However,

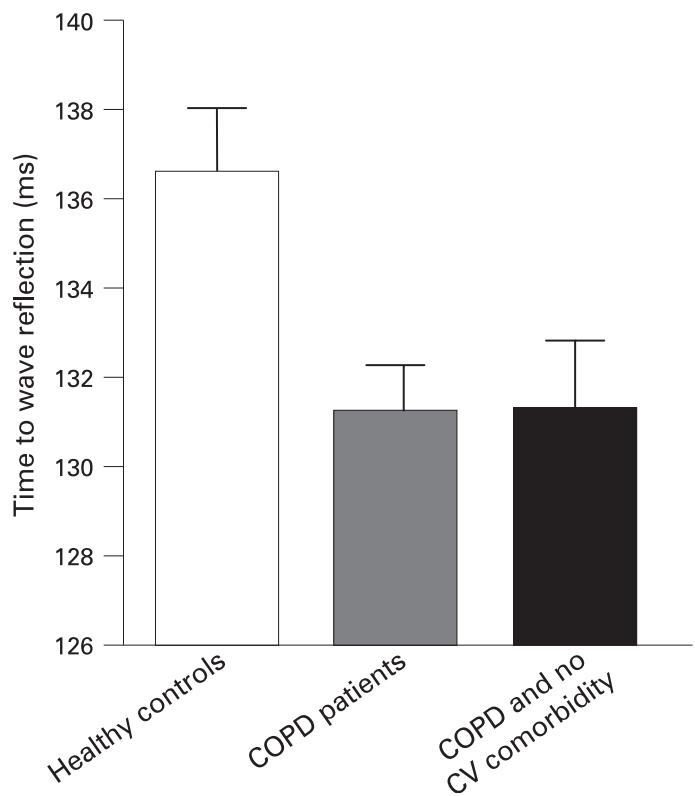

Figure 3 Mean time to wave reflection was reduced in patients with chronic obstructive pulmonary disease (COPD) $(p=0.004)$ and the subgroup of patients with COPD and no cardiovascular (CV) comorbidity $(p=0.03)$ compared with healthy controls matched for age and current smoking status.

local inflammation is associated with endothelial dysfunction in the pulmonary vasculature ${ }^{25}$ and it seems likely that similar processes may influence the extra-pulmonary vasculature. Using Salmonella typhus vaccination as a model of acute systemic inflammation, recent work suggests that inflammation can acutely increase aortic stiffness. ${ }^{26}$ This model has also been shown to transiently but profoundly alter systemic endothelial function. ${ }^{27}$ Interestingly, Kampus et al demonstrated that serum CRP concentrations are positively correlated with indices of arterial stiffness in healthy individuals. ${ }^{28}$

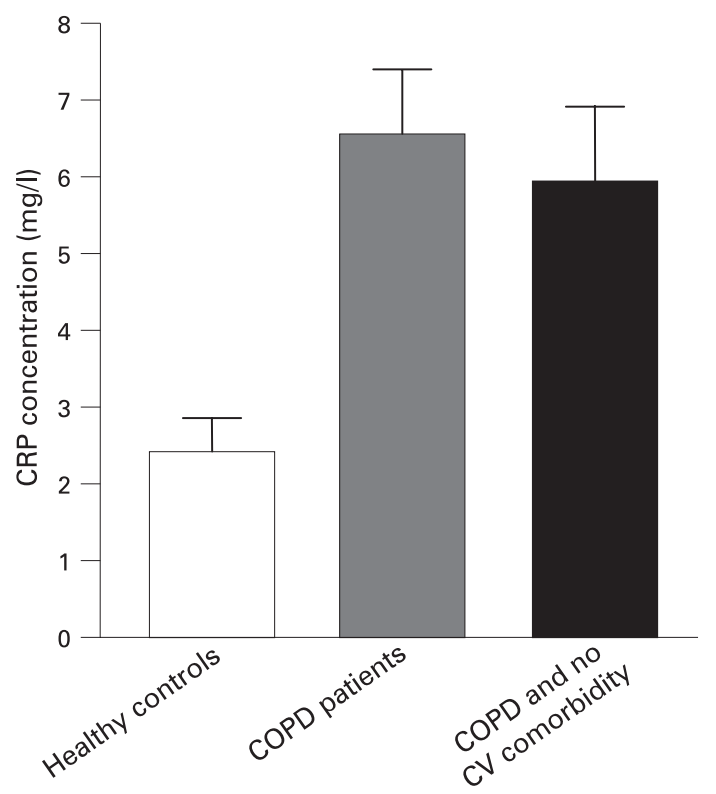

Figure 4 Mean serum $\mathrm{C}$ reactive protein (CRP) concentrations were increased in patients with chronic obstructive pulmonary disease (COPD) in the presence or absence of established cardiovascular (CV) comorbidity ( $p=0.001$ for both). 
Serum CRP concentrations were elevated in our patients and similar findings have been reported in other published COPD cohorts. $^{92}$ We hypothesise that systemic inflammation and vascular dysfunction may be responsible for our findings of increased blood pressure and arterial stiffness in COPD. The COPD patients in our cohort had a lower body mass index than healthy controls. This is a recognised feature of COPD and is likely to be a result of persistent bronchial and systemic inflammation. ${ }^{1}$ Serum CRP concentrations correlated with augmentation pressure in patients with COPD, but not in matched controls.

\section{Hypertension}

Our findings emphasise the need for better monitoring and treatment of traditional cardiovascular risk factors in patients with COPD. Based on the Joint National Committee on Prevention, Detection, Evaluation and Treatment of High Blood Pressure guidelines, ${ }^{30}$ two-thirds of our COPD population had blood pressures compatible with a diagnosis of hypertension, compared with just one-third of age matched controls. These findings are in keeping with a recently published prospective cohort study in which the prevalence of hypertension was approximately $50 \%$ in 5648 patients with COPD. ${ }^{11}$ Observational studies indicate that death from ischaemic heart disease and stroke increases linearly with blood pressure in all age groups. $^{32}$ For every $20 \mathrm{~mm} \mathrm{Hg}$ systolic or $10 \mathrm{~mm} \mathrm{Hg}$ diastolic increase in blood pressure, there is a doubling of cardiovascular mortality. A number of factors in addition to increased arterial stiffness may be responsible for the hypertension observed in patients with COPD.

\section{Study limitations}

One potential limitation in our study design is that many of the patients with COPD were recruited from a tertiary referral centre. This may have introduced some bias towards increased comorbidity that could potentially explain the observed differences between patients and controls. In order to counter this potential bias, we undertook a subgroup analysis in which patients with ischaemic heart disease and previously recognised cardiovascular risk factors were excluded. Differences in arterial stiffness and blood pressure persisted even when these patients were excluded.

While patients with COPD were characterised carefully with spirometry and reversibility, lung function was not measured in the healthy control group. It is possible that a minority of asymptomatic smokers had undiagnosed airways obstruction, although if anything inclusion of these subjects would be likely to reduce any important differences between the two groups. While we carefully matched patients with controls to ensure an equal balance of current smokers and ex-smokers to avoid the well established vascular and inflammatory effects of active smoking, there were differences in the number of pack years smoked between the cohorts. It is difficult to control fully for the cumulative effects of cigarette smoke in a case control study, as an abnormal inflammatory response in the lungs to cigarette smoke is central to the pathogenesis of COPD. Cigarette smoke has a well documented acute effect on blood pressure and arterial stiffness ${ }^{33}$ and both are increased in current smokers compared with non-smokers. ${ }^{34}$ However, a relationship between arterial stiffness or blood pressure and the magnitude of previous cigarette exposure (pack years) has not been established. We found no association between pack years smoked and any of the vascular parameters measured, and do not believe that small differences in past cigarette exposure account for the reported differences in vascular function between COPD patients and controls.

\section{CONCLUSIONS}

Patients with COPD have increased arterial stiffness, blood pressure and systemic inflammation. We hypothesise that systemic inflammation and vascular dysfunction may explain the excess cardiovascular morbidity and mortality associated with COPD. Further research into the mechanisms responsible for the increase in arterial stiffness is warranted. Increased awareness and targeted treatment of cardiovascular risk and hypertension has the potential to reduce morbidity and improve prognosis in COPD.

Acknowledgements: We would like to thank the Clinical Research Facility, Royal Infirmary Edinburgh, for their assistance with the studies.

Funding: The studies are supported by a National Institute of Health Grant (RFA-HL02-005) and a Programme Development Grant from the Chief Scientists Office, Scotland. NLM is supported by a Michael Davies Research Fellowship from the British Cardiac Society.

Competing interests: None

Ethics approval: All studies were performed with local ethics approval.

\section{REFERENCES}

1. Agusti AG. Systemic effects of chronic obstructive pulmonary disease. Proc Am Thorac Soc 2005:2:367-70.

2. Sin DD, Man SFP. Chronic obstructive pulmonary disease as a risk factor for cardiovascular morbidity and mortality. Proc Am Thorac Soc 2005;2:8-11.

3. Jousilahti $\mathbf{P}$, Vartiainen E, Tuomilehto J, et al. Symptoms of chronic bronchitis and the risk of coronary disease. Lancet 1996;348:567-72.

4. Camilli AE, Robbins DR, Lebowitz MD. Death certificate reporting of confirmed airways obstructive disease. Am J Epidemiol 1991;133:795-800.

5. Friedman GD, Klatsky AL, Siegelaub AB. Lung function and risk of myocardial infarction and sudden cardiac death. N Engl J Med 1976;294:1071-5.

6. MacNee W. Pathogenesis of chronic obstructive pulmonary disease. Proc Am Thorac Soc 2005;2:258-66.

7. Agusti AG, Noguera A, Sauleda J, et al. Systemic effects of chronic obstructive pulmonary disease. Eur Respir J 2003;21:347-60.

8. Ross R. Atherosclerosis—An inflammatory disease. N Engl J Med 1999;340:115-26

9. Sin DD, Man SF. Why are patients with chronic obstructive pulmonary disease at increased risk of cardiovascular diseases? The potential role of systemic inflammation in chronic obstructive pulmonary disease. Circulation 2003:107:1514-19.

10. Weissberg P. Mechanisms modifying atherosclerotic disease_-from lipids to vascular biology. Atherosclerosis 1999;147:S3-10.

11. McEniery CM, Yasmin, Hall IR, et al. Normal vascular aging: Differential effects on wave reflection and aortic pulse wave velocity: The Anglo-Cardiff Collaborative Trial (ACCT). J Am Coll Cardiol 2005:46:1753-60.

12. Laurent $\mathbf{S}$, Boutouyrie $\mathrm{P}, \mathrm{Asmar} \mathrm{R}$, et al. Aortic stiffness is an independent predictor of all-cause and cardiovascular mortality in hypertensive patients. Hypertension 2001;37:1236-41.

13. Zureik M, Benetos A, Neukirch C, et al. Reduced pulmonary function is associated with central arterial stiffness in men. Am J Respir Crit Care Med 2001:164:2181-5.

14. Celli BR, MacNee W, Agusti A, et al. Standards for the diagnosis and treatment of patients with COPD: a summary of the ATS/ERS position paper. Eur Respir J 2004:23:932-46.

15. Mackenzie IS, Wilkinson IB, Cockcroft JR. Assessment of arterial stiffness in clinical practice. OJM 2002;95:67-74.

16. Wilkinson IB, MacCallum H, Flint $L$, et al. The influence of heart rate on augmentation index and central arterial pressure in humans. J Physiol 2000;525:263-70.

17. Williams B, Lacy PS, Thom SM, et al. Differential impact of blood pressure-lowering drugs on central aortic pressure and clinical outcomes: principal results of the Conduit Artery Function Evaluation (CAFE) study. Circulation 2006;113:1213-25.

18. Weber T, Auer J, O'Rourke MF, et al. Arterial stiffness, wave reflections, and the risk of coronary artery disease. Circulation 2004;109:184-9.

19. Just M, Monso E, Ribera M, et al. Relationships between lung function, smoking and morphology of dermal elastic fibres. Exp Dermatol 2005;14:744-51.

20. Stockley RA. Neutrophils and protease/antiprotease imbalance. Am J Respir Crit Care Med 1999;160:49S-52.

21. Newman AB, Naydeck BL, Sutton-Tyrrell K, et al. Coronary artery calcification in older adults to age 99: Prevalence and risk factors. Circulation 2001:104:2679-84.

22. Wilkinson IB, Hall IR, MacCallum H, et al. Pulse-wave analysis: Clinical evaluation of a non-invasive, widely applicable method for assessing endothelial function. Arterioscler Thromb Vasc Biol 2002;22:147-52. 
23. Newby DE, McLeod AL, Uren NG, et al. Impaired coronary tissue plasminogen activator release is associated with coronary atherosclerosis and cigarette smoking: direct link between endothelial dysfunction and atherothrombosis. Circulation 2001:103:1936-41.

24. Newby DE, Wright RA, Labinjoh C, et al. Endothelial dysfunction, impaired endogenous fibrinolysis, and cigarette smoking: a mechanism for arterial thrombosis and myocardial infarction. Circulation 1999;99:1411-15.

25. Peinado VI, Barbera JA, Abate P, et al. Inflammatory reaction in pulmonary muscular arteries of patients with mild chronic obstructive pulmonary disease. Am J Respir Crit Care Med 1999;159:1605-11.

26. Vlachopoulos C, Dima I, Aznaouridis K, et al. Acute systemic inflammation increases arterial stiffness and decreases wave reflections in healthy individuals. Circulation 2005;112:2193-200

27. Hingorani AD, Cross J, Kharbanda RK, et al. Acute systemic inflammation impairs endothelium-dependent dilatation in humans. Circulation 2000;102:994-9.

28. Kampus $\mathbf{P}$, Kals J, Ristimae T, et al. High-sensitivity C-reactive protein affects central haemodynamics and augmentation index in apparently healthy persons. J Hypertens 2004;22:1133-9.
29. de Torres JP, Cordoba-Lanus E, Lopez-Aguilar C, et al. C-reactive protein levels and clinically important predictive outcomes in stable COPD patients. Eur Respir $J$ 2006;27:902-7.

30. Chobanian AV, Bakris GL, Black HR, et al. Seventh Report of the Joint National Committee on Prevention, Detection, Evaluation, and Treatment of High Blood Pressure. Hypertension 2003;42:1206-52.

31. Huiart L, Ernst P, Suissa S. Cardiovascular morbidity and mortality in COPD. Chest 2005;128:2640-6.

32. Lewington $\mathbf{S}$, Clarke R, Qizilbash $\mathrm{N}$, et al. Age-specific relevance of usual blood pressure to vascular mortality: a meta-analysis of individual data for one million adults in 61 prospective studies. Lancet 2002;360:1903-13.

33. Kool MJ, Hoeks AP, Struijker Boudier HA, et al. Short- and long-term effects of smoking on arterial wall properties in habitual smokers. J Am Coll Cardiol 1993;22:1881-6.

34. Jatoi NA, Jerrard-Dunne P, Feely J, et al. Impact of smoking and smoking cessation on arterial stiffness and aortic wave reflection in hypertension. Hypertension 2007:49:981-5.

35. Smith JC, Page MD, John R, et al. Augmentation of central arterial pressure in mild primary hyperparathyroidism. J Clin Endocrinol Metab 2000;85:3515-19.

\section{Lung alert}

\section{HIF1 $\alpha$ in hypoxia}

Conjugation of small ubiquitin-related modifier proteins (SUMO) to form larger substrates has been implicated in the regulation of multiple cellular processes in mammals including transcription, cell signalling and cancer progression. This conjugation is a dynamic process that can be reversed by a family of Sentrin/SUMO-specific proteases (SENPs).

This study investigated the physiological consequences of SUMOylation and deSUMOylation with particular regard to hypoxia-inducible factor $1 \alpha(\mathrm{HIF} 1 \alpha)$ and SENP1. A series of experiments were undertaken using SENP1 (a nuclear SUMO protease) knockout mice (SENP1 ${ }^{-\prime-}$ ). SENP1 was chosen as earlier studies implicated SENP1 mutations in early fetal death in mice and SENP1 is found in humans.

By comparing SENP1 $1^{-/-}$and SENP1 wild-type mice (SENP1 ${ }^{-/+}$), it was established that mid gestation fetal death occurred secondary to severe fetal anaemia because of deficient erythropoietin production. Erythropoietin production was subsequently linked with the stability of HIF1 $\alpha$, a process that was directly influenced by the presence of SENP1. Hypoxiainduced SUMOylation and subsequent degradation of HIF1 $\alpha$ is increased in SENP1 ${ }^{-1-}$ mice compared with wild-type mice, which suggests that SENP1 is essential to prevent degradation of HIF1 $\alpha$ in response to hypoxia. Further experimentation showed that the degradation of HIF1 $\alpha$ was through a Von Hippel-Lindau and ubiquitin/proteasome dependent mechanism.

This study highlights the important biological consequences that SUMOylation and deSUMOylation can have in mammals. In particular, the presence of SENP1 is essential to stabilise HIF $1 \alpha$ in response to hypoxia for prevention of early fetal death in mice. The study does not identify why hypoxia causes SUMOylation of HIF1 $\alpha$ and further studies are required to establish the nature of this.

- Cheng J, Kang X, Zhang S, et al. SUMO-specific protease 1 is essential for stabilisation of HIF1 $\alpha$ during hypoxia. Cell 2007;131:58495

\section{Kris McLaughlin}

Correspondence to: K McLaughlin, Princess Alexandra Hospital, Brisbane, Queensland, Australia; krismcl@doctors.org.uk 